\title{
Meta
}

Journal des traducteurs

Translators' Journal

\section{Grass, T. (2002) : Quoi ! Vous voulez traduire "Goethe "? Essai sur la traduction des noms propres allemands-français, Berne, Peter Lang, coll. « Travaux interdisciplinaires et plurilingues en langues étrangères appliquées », 296 p.}

\section{Georges Kleiber}

Volume 48, numéro 4, décembre 2003

URI : https://id.erudit.org/iderudit/008738ar

DOI : https://doi.org/10.7202/008738ar

Aller au sommaire du numéro

Éditeur(s)

Les Presses de l'Université de Montréal

ISSN

0026-0452 (imprimé)

1492-1421 (numérique)

Découvrir la revue

Citer ce compte rendu

Kleiber, G. (2003). Compte rendu de [Grass, T. (2002) : Quoi! Vous voulez traduire "Goethe " ? Essai sur la traduction des noms propres

allemands-français, Berne, Peter Lang, coll. « Travaux interdisciplinaires et plurilingues en langues étrangères appliquées ", 296 p.] Meta, 48(4), 611-614. https://doi.org/10.7202/008738ar d'utilisation que vous pouvez consulter en ligne. 
politico-économique est également mis en relief par Pym (1992), tout comme par Nord (1991) qui inclut dans son orientation des facteurs textuels, mais également des causes situationnelles, médiatiques, communicatives et fonctionnelles.

Ce livre nous présente en fait un immense et fascinant panorama mondial des diverses orientations théoriques et pratiques de la traductologie par la description et l'explicitation de l'évolution des mèmes étudiés à travers le temps et l'espace. Le chapitre 3 s'attache à la question des mèmes et de la norme en analysant le concept de norme de traduction et en explicitant une classification des diverses catégories normatives. Le chapitre 4 explicite les mèmes des stratégies de traduction dans leurs applications pratiques. L'auteur utilise un texte publicitaire pour mettre en relief les diverses stratégies utilisées, notamment les stratégies syntaxiques (traduction littérale, emprunt, calque, transposition, changement d'unité, changement de structure de groupe, changement de structure de proposition, changement de structure de phrase, changement de cohésion, changement de niveau, changement de schème). Le chapitre 5 reprend le modèle basé sur Popper en illustrant par des exemples des diverses phases du schéma décrit. Le chapitre 6 donne les aspects du développement de la compétence en traduction en mettant en relief l'importance des mèmes et de leur signification dans les stratégies de formation et de production. Le chapitre 7 centre l'attention sur la question de l'éthique de la traduction. Le livre se termine par un «Épilogue » qui réclame la mise en pratique sur tout le matériel présenté de la phase préconisée de l'élimination des erreurs (EE) dans la théorie poppérienne de la traduction. Soulignons encore l'importance des références bibliographiques et l'utilité de l'index des auteurs cités ainsi que l'index des sujets traités.

Le livre d'Andrew Chesterman est un «must» pour tous les traducteurs et tout particulièrement pour les étudiants en traduction. Le livre est agréable à lire et sans prétention. C'est, à ne pas douter, un livre qui ouvre les portes de la connaissance théorique et pratique en présentant les multiples facettes de la traduction. C'est aussi un livre qui éclaire de façon pénétrante le vaste domaine de la théorie de la traduction, qui autorise toutes les remises en questions, qui organise la matière en ne préconisant que l'indiscutable démarche poppérienne de l'élimination des erreurs, procédé des plus utiles et des plus fertiles pour la réflexion, la formation de l'esprit et le processus de découvertes scientifiques. C'est encore un livre qui aborde les questions essentielles en les reliant à l'ensemble du domaine, qui montre qu'il faut donner la priorité à une formation pleine, qui explicite un cheminement logique et indispensable dans une démarche réflexive, qui alimente la discussion et permet à chacun de tracer sa voie, qui donne une remise en question de tout, bref qui fait amplement et sainement réfléchir. C'est en fait un magnifique guide pour un séminaire sur la théorie de la traduction. Il est à recommander à tous les responsables de cursus dans les divers instituts et écoles de traduction. La formation et la pratique ont tout à y gagner.

André Clas

Université de Montréal, Montréal, Canada

Grass, T. (2002): Quoi! Vous voulez traduire "Goethe»? Essai sur la traduction des noms propres allemands-français, Berne, Peter Lang, coll. «Travaux interdisciplinaires et plurilingues en langues étrangères appliquées », 296 p.

Après l'ouvrage récent de M. Ballard (2001, Le nom propre en traduction, Paris, Ophrys), voici un nouvel ouvrage sur la traduction des noms propres, dont la particularité est de se centrer sur la traduction des noms propres allemands-français, alors que celui de Ballard porte essentiellement sur la traduction anglais-français. L'exclamation du titre rappelle plaisamment que, généralement, les théoriciens des noms propres font de l'intraduisibilité une 
propriété spécifique de ce type de dénominations qui refléterait leur absence ou du moins la ténuité de leur sens. Dans la pratique, on sait bien qu'il en est autrement: on est très vite confronté au problème de savoir s'il faut traduire tel nom propre ou non et si oui comment il faut le faire. Et ceci que ce soient des noms de personnes comme Aristote, qui devient en allemand Aristoteles, des noms de lieux où - les Alsaciens le savent bien - la Forêt-Noire en face est der Schwarzwald, ou encore des noms d'œuvres d'art (la Joconde, c'est en allemand die Mona Lisa), des monuments (la tour Eiffel, c'est der Eiffelturm), etc. Bref, comme le souligne l'auteur dans son introduction (p.1), «il n'apparait pas si aberrant de prendre en considération l'importance des noms propres en traduction et la nécessité d'envisager un traitement spécifique de cette classe de mots au niveau lexical». Ceci d'autant plus que dans un corpus journalistique les noms propres représentent un dixième environ de l'ensemble des mots (p. 2). Mais comment faire?

Deux types de problèmes se posent immédiatement:

(i) celui de la délimitation des noms propres : qu'est-ce qu'on retient comme nom propre pour objet d'étude?

(ii) comment classer en sous-catégories les noms propres retenus? En somme, quelle subdivision adopter pour leur reconnaissance et traitement dans le cadre traductif adopté?

La première question suppose que l'on se penche sur la définition des noms propres, la seconde que l'on élabore une typologie des noms propres qui soit efficace. Le chapitre I règle la première, les autres chapitres, surtout à partir du chapitre III, s'occupent de la seconde.

Le chapitre I («Qu'est-ce qu’un nom propre?», p. 11-39) répond à la première en effectuant un parcours assez complet à travers le temps et les théories sémantiques du nom propre. Thierry Grass envisage successivement le traitement du nom propre dans les grammaires, la question centrale et si complexe de la référence des noms propres, le problème de la notoriété, celui des rapports entre noms propres et encyclopédie, noms propres et terminologie, le passage du nom propre au nom commun (il préfère dans ce cas le terme d'éponyme au classique antonomase, p. 33) et celui, inverse, du nom commun au nom propre. Au terme de son investigation, il propose une définition du nom propre qui en fait «un nom associé primairement à un référent individualisé. Le référent est un être vivant ou divin, un lieu, une œuvre humaine ou encore un événement unique, son existence est culturellement notoire, c'est-à-dire attestée dans les faits, dans le mythe ou dans la fiction» (p. 38). Définition, soulignons-le, dont le but est avant tout «utilitariste» et qu'il ne faudrait donc pas juger en théoricien du nom propre. Du point de vue théorique, c'est la caractérisation fournie page 36 de l'opposition nom commun-nom propre qui peut être mise en avant par son côté stimulant: «Le nom commun entretient une relation primaire avec le concept et secondaire avec le référent, alors que le nom propre entretient une relation primaire avec le référent et secondaire avec le concept.» La définition "pratique» a pour but de permettre à Thierry Grass de retenir les noms propres qu'il veut ou qu'il faut retenir traductorialement parlant. Le primairement de la définition (associé primairement à un référent individualisé) est assez commode en ce qu'il autorise à considérer un Français, par exemple, comme nom propre (p. 19), ce qui peut se comprendre dans une perspective traduisante - il faut bien le traduire! - mais qui s'avère bien vite difficilement compatible avec toute définition homogène des noms propres. Par ailleurs, la notion de référent individualisé ne se laisse pas manipuler sans difficultés. Il est plutôt surprenant de constater que sur la base de la définition postulée, Thierry Grass reconnaît comme noms propres Yahvé, Jésus, Allah, mais non Dieu, celui-ci étant, selon lui (p. 38), un nom qui renvoie «primaire ment à un concept sous forme d'attribut». Exit de la même manière des noms de technologie nouvelle comme Internet ou World Wide Web.

Le chapitre II est consacré aux «perspectives diachronique, pragmatique et computationnelle en onomastique» (p. 41-79). Il vise, d'une part, à montrer la différence d'approche selon que le point de vue est diachronique ou classificateur comme dans les analyses prag- 
matique et computationnelle et, d'autre part, à effectuer une synthèse conduisant à une classification des noms propres utilisable pour l'objectif de traduction fixé. Le lecteur trouvera de nombreux renseignements sur les traitements des noms propres en Allemagne, notamment sur la typologie pragmatique (c'est-à-dire à partir du référent) des noms propres de G. Bauer (1998, Namenkunde des Deutschen, Berlin, Germanistiche Lehrbuchsammlung, Band 21) qui distingue cinq catégories, dont s'inspirera Thierry Grass: les anthroponymes (êtres humains), les toponymes (ayant l'espace où vit l'être humain comme référent, les ergonymes (ayant les objets élaborés par l'être humain pour référent), comme par exemple le journal Der Spiegel), les praxonymes (ayant les activités accomplies par l'être humain pour référent, comme par exemple la Révolution française) et les phénonymes (ayant les événements ne dépendant pas de l'être humain pour référent, comme par exemple le cyclone Mitch ou, chose plus surprenante, Vénus, la planète). Du point de vue informatique, c'est la reconnaissance automatique des noms propres et surtout la reconnaissance de leur catégorisation qui sont au centre du débat. Thierry Grass introduit à cet effet une théorie syntaxico-sémantique propre au traitement informatique des noms propres, la théorie des classes d'objets de Gaston Gross, qui permet d'envisager le nom propre non pas isolément, mais dans un réseau de cooccurrences lexicales.

Le chapitre IIII ( «Le nom propre en traduction», p. 81-154) prépare directement l'analyse en termes de traduction des différents types de nom propre qui formera la deuxième partie de l'ouvrage. Dans ce chapitre III, l'auteur présente, d'une part, les particularités syntaxiques contrastives des noms propres en allemand et en français et, d'autre part, les différentes méthodes ou techniques de traduction des noms propres et le projet Prolex de traitement automatique des noms propres, dont Thierry Grass est membre. Il ressort de son enquête que «l'essentiel est [...] de rentrer les noms propres les plus connus qui sont aussi ceux $[. .$.$] dont les formes varient le plus entre la langue source et la langue cible» (p. 154).$ Les autres, ceux qui ne seront pas codés, doivent pouvoir être repérés comme noms propres, à l'aide de différents indices qui seront exposés dans les chapitres suivants et grâce à un traitement détaillé et le plus exhaustif possible des autres lexèmes, car, comme le souligne Thierry Grass, "plus l'analyse d'un texte par un dictionnaire de langue générale est précise, plus les mots inconnus se révèlent être des noms propres» (p. 154).

Les quatre derniers chapitres, consacrés respectivement aux anthroponymes (ch. IV), toponymes (chapitre V), ergonymes (chapitre VI) et pragmonymes (chapitre VII), classe constituée par Thierry Grass en rassemblant les praxonymes et phénonymes de Bauer (voir cidessus), forment la partie la plus importante de l'ouvrage, la plus riche, qui constitue sans aucun doute un apport précieux, et sur de nombreux points novateur, pour la problématique de la traduction des noms propres allemands-français. Thierry Grass détaille les différents problèmes que posent les quatre classes de noms propres, elles-mêmes bien souvent encore subdivisées en sous-classes, énumère les solutions retenues, présente des analyses en traitement automatique du langage (voir par exemple p. 166-167, le transducteur à états finis pour analyser les noms propres de famille), maitrise la délicate question du genre et du nombre (voir pour les toponymes, p. 179-184) et celle, tout aussi complexe, de la détermination. Surtout intéressant se révèle le recours, annoncé dans le chapitre III préparatoire, aux classes d'objets pour coder les noms propres, même s'il n'est pas toujours facile à effectuer, comme l'auteur l'a lui-même souligné (p. 139). L'aide des classes d'objet permet dans les quatre grands types conceptuels de noms propres analysés de mettre en relief des structures et schèmes syntaxiques caractéristiques, fort utiles pour une gestion automatique des noms propres en traduction. La richesse de ces quatre chapitres nous interdit d'entrer dans le détail des données, des descriptions, des analyses et solutions proposées. On se contentera de signaler que les chapitres les plus originaux nous semblent être le chapitre VI et surtout le chapitre VIII, parce que les noms propres qu'ils rassemblent sont beaucoup moins connus que les plutôt familiers anthroponymes et toponymes et parce qu'ils donnent lieu à une 
exploitation syntaxique en termes de classes d'objets peut-être plus convaincante et plus ferme que celle réalisée avec les deux premiers types de noms propres.

Le bilan est donc positif et on comprend aisément qu'un tel ouvrage ait pour prolongement naturel, annoncé en conclusion, la parution d'un dictionnaire bilingue des noms propres. On comprendra aussi que les spécialistes de la sémantique des noms propres aient tout intérêt à mettre leur nez dans l'ouvrage: ils y trouveront à coup sûr du grain à moudre pour leur propre moulin. Sans doute pas là où Thierry Grass le pense, c'est-à-dire pas dans le chapitre I définitoire et sans doute pas dans la leçon sémantique d'un nom propre «beaucoup plus lourd de sens qu'il ne le paraissait» (p. 287). Mais beaucoup plus dans la présentation et illustration de noms propres diversifiés, regroupés en quelques grands types conceptuels, qui donnent à penser que la fameuse version faible du sens des noms propres promue aussi bien par des philosophes comme Geach que des linguistes comme Buyssens et Maurice Gross, et remise en selle récemment par Van Langendonck en 1999 ( «Neurolinguistic and syntactic evidence for basic level meaning in proper names», Functions of Language, 6-1, p. 95-138), mériterait vraiment qu'on la reprenne à la lumière des informations apportées par l'ouvrage de Thierry Grass.

Georges Kleiber

Université Marc Bloch, Strasbourg, France

\section{Pöchhacker, F. (2000): Dolmetschen. Konzeptuelle Grundlagen und deskriptive Untersuchungen, Tübingen, Stauffenburg Verlag, 336 p.}

Plus encore que le titre, très global, c'est le sous-titre qui, d'emblée, donne la visée et la mesure de l'ouvrage. L'auteur, d'ailleurs, souligne ce fait dans la préface qui retrace en quelque sorte le cheminement de l'ouvrage: partant de la notion de l'interprétation, ancrée dans son contexte historique, en passant par la "traductologie ${ }^{1}$ », et les fondements théoriques de l'interprétation au versant plus pratique du community interpreting avec ses multiples facettes jusqu'aux perspectives de cette science sans oublier pour autant les possibilités de recherche dans le domaine. Enfin, les recherches empiriques de l'auteur témoignent bien combien il s'est investi en la matière.

Voici quelle est la structure de cet ouvrage: la table des matières se compose de sept chapitres, d'un résumé en anglais pour chaque chapitre, d'une bibliographie et d'une annexe qui reproduit les questionnaires utilisés pour la recherche empirique. Les chapitres semblent suivre une certaine chronologie de l'évolution de l'interprétation sans pour autant l'indiquer expressément. L'importance accordée aux différents chapitres d'après leur taille est à peu près identique. Ce qui frappe d'emblée le lecteur de cet ouvrage, qui se veut également didactique, c'est qu'un index onomastique et thématique fait défaut.

En plus de couvrir les principaux aspects du champ de connaissances, qui est l'interprétation, les composantes de ce champ sont présentées de manière méthodique, dans un souci didactique, voire vulgarisateur. Nous trouvons les thèmes subdivisés en sous-thèmes et sous-sous-thèmes qui s'intègrent dans un ensemble cohérent. L'aspect «historique » de l'interprétation avec l'évolution de la notion d' "interprétation " au fil des siècles est brièvement esquissé et l'auteur, germanophone, ne pouvait guère - dans ce contexte - se limiter au seul champ de l'interprétation sans passer par la traduction. Car la notion allemande de Translation forgée par Otto Kade $(1963)^{2}$ comprend bien la transmission orale et écrite d'un récit ou texte d'une langue dans une autre.

En ce qui concerne l'histoire de l'interprétation, dans la bibliographie, pourtant bien fournie, le lecteur cherche en vain quelques ouvrages-clés qui ne devraient pas manquer dans ce contexte, d'autant plus que les données bibliographiques et leur analyse prennent 